ISSN: 2455-104X

Impact Factor: 4.950

Volume 4,Issue 1 (Jan-June) 2018, 60-66

Received: 21 June. 2018 ; Accepted: 28 June. 2018 ; Published: 07 July. 2018 ; Paper ID: IJRLS-1282

\title{
PROBLEMS AND RETRIEVAL OF INFORMATION IN DIGITAL ENVIRONMENT
}

\author{
SajiniPriya Natarajan ${ }^{1}$; K. M. Krishna ${ }^{2}$ \\ Research Scholar, Bharathiar University, Coimbatore, Tamil Nadu. ${ }^{1}$; \\ S K N Agriculture University, Jobner (India) ${ }^{2}$ \\ rsajpriya@gmail.com ${ }^{1}$; kmkskn@gmail.com ${ }^{2}$
}

\begin{abstract}
The present paper reports retrieval problems of information with changing digital environment. The users are more thoughtful to challenge with any new technological devices and their retrieval mechanism devices.There is need to study and explore user's problems with changing information learning system.
\end{abstract}

Keywords: Users retrieval Interface (URI), user's information retrieval mechanism, digital retrieval devices, Rewiring of Users mind, Limitations of retrieval mechanisms of users in digital learning environment.

\section{INTRODUCTION}

No doubt users play important role in retrieval of information in their learning system. Users retrieval mechanism needs better aptitude for learning devices and efficiency to handle for better use of information .It is known that the retrieval information mechanism of users are more dependent on creativity, concentration and skill of users mind to retrieve the desired information.In the traditional information retrieval systems, users are more thoughtful to search, select and discuss with the professionals to generate ideas into channels of discoveries, inventions and schools of thoughts, where as in the modern digital retrieval system, users will be more concerned with changing devices with knowledge skills and experience to search desired information to make school of thoughts ,however users are still habitual of old mechanism to retrieve any information in the learning system. The scope of earlier practices may dominate to some extent but users mind are now gradually switching into faster modern information retrieval mechanisms with the changing technological devices. The user's minds still need to equip with changing technological devices for the thoughtful retrievals of information.

\section{Objectives}

The various objectives of the present study are

- To know the various traditional retrieval mechanisms of information.

- To study the modern and future retrieval mechanisms of information with changing devices in digital learning system.

- To know the role of modern information retrieval devices: Internet and TV Channels, digital tablets, e learning /i learning system as source of information learning.

- To know the scope of tablet PC as future information learning system. 
- To know the role of users mind as retrieval mechanism of information handling.

- To know the limitations of user's retrieval mechanisms in digital information system

\section{User's Retrieval Interface (URI)}

The retrieval mechanisms of information much depends on the storage and minds of users responsible for creation and management of retrieval thoughts which are infinite, unlimited, and available in variable constraints. No doubt user's minds are also changing with contemporaneous developments of emerging technology which has almost metamorphosed the traditional retrieval system into digital retrieval learning system but there is still need to define thoughts of users mind with Usersretrieval Interface(URI ) , particularly in selection of information based on strings of information which are thoughtful, natural, imaginative and digitized in selection. The selection of information will be accurate when it is based on problems and capable to perform multitask in a fraction of time to multi users in various disciplines but there is need of intelligent retrieval system based on thoughts, experience and knowledge skill of multi requirements of users. Users mind are capable to asses any logic problems at any critical juncture to metamorphose Long Range Reference service into Short Range Reference services to the satisfactory level of users but it changes with people, age, experience, knowledge, sex, imagination, thoughts ,organizations, schools, societies, scientific communities, environment etc. The user's minds are capable to gather strings of archival thoughts in a fraction of seconds to solve any critical innovative problems.

The most important part of user's retrieval mechanisms is the selectivity of information based on problems and requirements of users to use further .It triggers the users mind to query any information access for further processing.

\section{Retrieval Mechanisms of information access}

No doubt, the existing traditional retrieval platforms will be metamorphosed into modern Digital devices like Internet/Intranet , computers, digital tablets, TV learning channels, LAN/WAN, Wi-Fi system etc. which will be used as retrieval mechanisms for information in digital environment. The two prominent retrieval mechanisms for information handling are:

- Traditional retrieval mechanisms of information access

- Modern retrieval mechanisms of information access

\section{Traditional retrieval mechanisms of information access}

There are various retrieval mechanisms of users in traditional system .The existence of such practices still makes viable source of retrieving information and widely used for information handling among scientific and non-scientific communities as source of learning.

These are

- Lectures, teachings as source of learning

- Schools, colleges, universities, organizations, research centers

- Scientific meetings like seminar / conferences / refresher courses/ symposiums

- Group discussion by the expertise/ queries

- Medical expertise interface

- Tacit knowledge interface

- Questionnaires

- Teaching communication knowledge

- Scientific observations / surveys / methods / experiments

- Use of sensory organs into logical values as decision making processes

- Libraries / archives / history / monuments

- Geophysical / geographical / biological / ecological events

- Religious / spiritual / educational thoughts 
- Disaster events like earthquakes, cyclones, floods, hurricanes, tsunamis, landslides, snowfalls, rains, global warming's / climatic changes as information retrieval mechanism to make frequent logical knowledge

- Mapping of entire thoughts as retrieval mechanism

- Activation/deactivation of mind with ages, experiences

- Role of creativity and concentration of users mind in retrieval of information

- Radio, letters correspondence

- Charts / slides/ herbariums, samples as source of learning etc

- Indexing / catalogue / classification schemes / Directory etc.

\section{Modern retrieval mechanisms of information access}

- Social networking groups like Face book, Twitter, Orcut,

- Yahoo groups LinkedIn as resource sharing

- Internet search engines / websites / portals / emails

- Online access facility

- Mobile Alert library service for e Resource Query with registration of institutional /users mobile numbers for document delivery system

- Online books and journals access facility

- $\quad$ Digital tablets, / e books reader / apps

- Cable networks, e learning packages, e tutorials

- $\quad$ CD / DVD audio visual system / pen drives /reprography / scanners, LCD projectors

- Library software's,/open source software's, soul, libsys, e granthalaya,koha, D space, GSDL, etc

- Open sources journals (www.doaj.org)

- Online Library membership

- $\quad$ OPAC / SDI, web Catalogue, web directory

- Internet / Internet access facilities

- User ID / Password

- IP Based Access

- Computers / Laptops / Tablets PC / pen drive /HDD / RAM /ROM / iPad

- E tutors, e teachers, e colleges, e schools of learning,

- $\quad$ E publishing /E presentation, /e counselors

- National digital repository

- Digital Charts / slides/ herbariums, samples as source of learning etc

- News channel /TV / Live cast / e newspapers

- $\quad$ E resource learning centre/digital repositories etc

- Digital learning devices Aakash tables, TV networks , online access

- $\quad \mathrm{LAN} / \mathrm{WAN} / \mathrm{Wi}-\mathrm{Fi}$ / satellite system , network servers 
- Different reading formats /MSWord / PNG, JPEG , JPG, PDF

- Operating systems internet explorer, Mozilla, opera, Netscape, Google chrome to download files / texts / antivirus etc

- Perception of eyes, earphones, speakers, headphone, microphone

- $\quad$ Chatting text / voice chats

- Virtual libraries, virtualresources, virtual e learning centers.

\section{Design of Query for retrieval mechanism in digital environment}

There is need to study the design of retrieval mechanisms of users in digital learning system. In this ,the role of users mind will certainly play an important role for creation and design of retrieval mechanisms which will be based on attitude and query of users profile or organization. No doubt, User's brains are well equipped and naturally organized receptors to evaluate understand and make inferences of critical problems of any queries. So, the concept of query is almost dependent on cognitive status of users mind as receptors to make thoughtful, useful, interactive, selective, creative, logical, innovative and imaginative events of multitasking problems of multi users.

- The user's information query in digital environment will depends on various constraints

- $\quad$ Type of information query

- $\quad$ Types of users

- Gender of users

- Age of users age groups child / adolescence /old

- Psychology of users

- Education of users

- $\quad$ Language to express query

- Knowledge of users to ask query

- Consciousness /unconsciousness of users

- $\quad$ Physical mind of users to ask query

- Professional users

- Interdisciplinary professionals

- Others users searching various information

- $\quad$ Skilled / Unskilled users for selective query

- Lay man search methods

- Lack of knowledge in keywords or subject expertise

- $\quad$ Language barriers

- $\quad$ Spelling problems

- Meta data problems

- Imagination and memory and intuition

- Noise communication (filtered/unfiltered information)

- Users minds towards strings of archival thoughts

- Mapping of users thoughts with changing technologies 
- Expression of multi thoughts of multi users

- Social networking's

- Internet connectivity, Wi-Fi system and its speed,

- Use of different reading formats / update Operating systems /virus attack / LAN/WAN

- Different operating system like window xp, vista, window 7 , Linux, Unix

- Orientation programs to get aware of digital devices

- Awareness of changing hardware / software devices

- Computer literacy of users

- Online subject specialists for users help.

\section{User's retrieval devices and applications used for information access}

There are various technological devices used for retrieval of information as system of learning. Users are now habitual to make use of such changing technological devices in their learning system. In near future, the new modern retrieval devices will occupy the traditional retrieval mechanisms to share or to access selective information. The devices used for retrieval of information are hardware ,software's, internet, computers, digital tablets, TV channels ,Wi-Fi, LAN/WAN , e learning system , CD / DVD , databases pen drives , e books , and its e book reader, reading formats like PDF, word, PNG, JPG, JPEG , EXCELL, PAGE MAKER , HTML, XML, etc to access any information ..The different browsers like Opera, Mozilla, Internet explorer, Netscape are very important tools to download / retrieve any files or text as source of learning likewise the different operating system like window xp, 7, vista ,Linux, Unix , androids are useful software devices of computers / tablets as retrieval mechanism of users .

The computers can't observe the problems to assess or evaluate or solve the problems and make its own decision after all users minds are helpful to make its own decision and capable of learning system to map out the information archives. In digital learning system, the mind will certainly play vital role in decision making process.

The best use of information based on logic values can help but can't be solution of users retrieval mechanism for inferences leading to innovative ideas /discoveries which ultimately useful to social / scientific communities and people of the society. After all users minds are well equipped with knowing the existence of natural problems and makes its own logical thoughts to achieve its goal. The Sensory receptors which acts like input devices such as eyes, ears serve are helpful in making best use of users mind leading to thoughtful activity, the inputs device of computers are keyboard, mouse, CPU, monitor are concerned, they not only performs routine works but retrieves many manipulations, many managerial tasks at critical juncture but they are not thoughtful to make conclusionof usersinformation system. It has been seen many times that there has been a regular cyber-attack on the internet server, these software must be fully protected specially from own software operating system .The hacking of official website makes hindrance in retrieve invaluable information in retrieval mechanism such things must cleared with protection acts and steps to use Intranet services as security devices /solutions like intruder detection system , firewall identification system, digital signatures and security networks must be able to tackle cyber-attacks and suitable laws must be framed out. Optimum use of internet server as retrieval mechanism must be adopted to minimum extent.

No doubt, we are entering into new era of learning system where the natural minds of users will be replaced by digital minds of learning with new technological devices like Internet, computers, laptops and tablets. Computers and TVnetworks devices which are capable to adhere human minds with new technological devices in digital learning system. In order to adhere knowledge share, nurture users of different organizations there is need of online e -resources services , websites , portals , web links, gateways, internet / intranet servers, IP address, machine login numbers on networks , resource sharing, emails , social networking's sites etc. In future, there will be only digital based information sources which can be accessed by each and every user of schools, colleges, universities and other organizations irrespective of space and time factors. In present scenario , the information which are available in traditional formats or platforms will be metamorphosed into digital mode like e books, e journals , e publishers ,e teachers , e subjects, e tutorials ,e learning resource centre's that can be available on sale or on consortium price. In future the traditional devices will be completely replaced into digital mode to adhere new modern learning system. The country like India where such cheapest tablets like Aakashwill be used as learning device have lot of scope of for schools, colleges and universities located in remote places / villages.The NCERT textbooks have 
been digitized and are available free on portals. Likewise, there will be digitization of more books to provide free on portals to students of colleges and universities, institutions. There will be large set up of e schools, e colleges; e universities to get distance education easily on digital tablets or TV networks. We will have adequate number of e learning, e tutorials studies available on portals or Tata sky TV and other networks .

\section{Role of users mind as retrieval mechanisms}

- Relation between thoughts and users mind

- $\quad$ Role of Memory, imagination and intuition as learning system

- $\quad$ Rewiring of Users mind

\section{Relation between thoughts and users mind}

In digital learning system the users will play an important role to adhere digital learning system Users minds are most thoughtful which helps to retrieve any logical method or useful information which are based on time based daily, weekly , monthly, yearly or even in fraction of time some are based on problems, thoughts, events to make useful information, so retrieval of users thoughts vary from people, experience, age, sex, environment and changing technological devices

The users brains are responsible for thoughts and making creativity information access . The right brains which accounts for creativity, random, intuitive, holistic, subjective thinking whereas left brains are for logical, sequential, rational analytical and objective looking to the first left brains more maybe logical or may not success in information access but the human catchy eyes are very much responsible for information access or retrieval more faster. User's thoughts are more perceptive, cognitive, and interactive in retrieval mechanism of information access.So, the Aesthetic sense is required for attaining knowledge the memory, intelligence and logic are important for user's brain.

Whatever the future digital systems are developing towards information storage and retrieval systems, the users minds are in tuned with strings of thoughts which are infinite, unlimited creative in thinking which comes to reality of new world of philosophy as new information learning.

\section{Role of Memory, imagination and intuition as learning system}

The memory and Concept of forget memories are constant encoding process within the brain. We know similar parts of the brain becomes active when someone is talking about mistake or real information .Interpretation of information constructed from diverse sources including visual and other sensual information as well as more general knowledge and past experience. Many different parts of the brain are involved in forming memories. The brain edits the information, we take in even before it is laid down as memory and there is more editing as the memory is formed. One hypothesis, the part of the brain responsible from processing and storing memories is very sensitive to human stress system .A small group of cognitive scientists is arguing that users of academic institutions could take far more advantages of this same bottom up ability called perpetual learning. According to kellman (PJ) any theory of how we learn presupposes perceptual knowledge that we know which facts are relevant that we know what to look for makes efficient.Scientists have long known the brain registers subtle pattern subconsciously well before a person knows he or she is learning .According to Sloman (S) of cognitive scientists at Brown University Rhode Island, "Once the brain has goal in mind, it tunes the perceptual system to search the environment for relevant clues in time eyes, ear and nose learn to isolate those signs and dismiss irrelevant information sharpening thinking of users mind.

\section{Rewiring of Users mind}

One at the age of 70 cannot stop thing about new topics one has go through training to make brain more sharper with cognitive minds can trigger better use of mind in retrieval mechanism of information access, loss of memory is usual phenomenon with Age, Sex, physical constraints, but neuroscientists are able to train users minds as human body which can enhance the rate of working, training which could reverse the cognitive decline of brain. Good brains exercises are cleverly designed to concentrate long periods of mental activity into short periods of intense training after 20s most people set their learning on cruise mode and try to confine their knowledge acquisition to narrow ranges.

Scientists involved in training focus their attention on the cortex which is part of higher learning in the enriched environment for human would include physical activity continues learning strong social life and deep interest in art.Computers like users 
can learn, but when Google tries to fill our search box based on a few keystrokes or our iPhone predicts words as you type a text massage, it's only a narrow mimicry of what user's brain is capable. The challenge in training computers to behave like a user's brain is technological and physiological testing the limits of Computers and Brain Sciences.

\section{Limitations of user's retrieval mechanism in digital environment}

users brain is processing in parallelism which can perform multi task and tests at a time but restricts in computer system which can only perform with the help of duplicate hardware's or devices and has less retention power to make innovative thought etc. Whateverthe retrieval devices are made the users retrieved thoughts are not well equipped with changing retrieval devicesof learning system.

\section{Authencity of portals / websites / web pages / search engines / web information}

PC tablets restricts to research and development of scientific institutions or organizations where large equipment's or on site experiments are involved. But will be extensive useful to the fields of many Sciences, Social sciences, Arts and other disciplines.

\section{Conclusions}

Overall present study reveals that there is still need to study the attitude of users in retrieval mechanism with changing technological devices and networking platforms. In different schools of thoughts users are also learning with variable digital devices or portal platforms which are much dependent on variable retrievals of knowledge, awareness, experience , age , sex , and other learning factors .The different learning portal platform as retrieval mechanism of information needs Authencity .

\section{REFERENCES}

[1]. Krishna K M (2007) User retrieval problems during searching of specific information through Internet: a study analysis. SRELS JL of Information Management, 44,(1)P77-82

[2]. Krishna K M (2010) Digital information access and its impact on reading habit of users SRELS JL of Information Management, 47( 3 ), 289-295

[3]. Krishna K M (2011) Relevance of LIS professional networking: study and analysis of orcut social group SRELS Journal of Information Management Volume: 48( 5) ,545-550 